\title{
High-fat-diet-induced inflammation depresses the appetite of blunt snout bream (Megalobrama amblycephala) through the transcriptional regulation of leptin/mammalian target of rapamycin
}

\author{
Yong-Jun Dai, Guang-Zhen Jiang, Xiang-Yang Yuan and Wen-Bin Liu* \\ Key Laboratory of Aquatic Nutrition and Feed Science of Jiangsu Province, College of Animal Science and Technology, \\ Nanjing Agricultural University, No. 1 Weigang Road, Nanjing 210095, Jiangsu Province, People's Republic of China \\ (Submitted 22 April 2018 - Final revision received 7 August 2018 - Accepted 3 September 2018)
}

\begin{abstract}
The aim of this article was to investigate the mechanism of appetite suppression induced by high-fat diets (HFD) in blunt snout bream (Megalobrama amblycephala). Fish (average initial weight 40.0 ( $\operatorname{sem~} 0.35$ ) g) were fed diets with two fat levels (6 and $11 \%$ ) with four replicates. HFD feeding for $30 \mathrm{~d}$ could significantly increase the weight gain rate, but feeding for $60 \mathrm{~d}$ cannot. Food intake of $M$. amblycephala began to decline significantly in fish fed the HFD for $48 \mathrm{~d}$. HFD feeding for $60 \mathrm{~d}$ significantly reduced the expression of neuropeptide $\mathrm{Y}$ and elevated the expression of cocaine- and amphetamine-regulated transcript (CART), actions both in favour of suppression of appetite. The activation of fatty acid sensing was partly responsible for the weakened appetite. In addition, inflammatory factors induced by the HFD may be involved in the regulation of appetite by increasing the secretion of leptin and then activating the mammalian target of rapamycin (mTOR). Lipopolysaccharide (LPS, $2.0 \mathrm{mg} / \mathrm{kg}$ of fish weight) was administered to induce inflammation, and sampling was performed after 3, 6, 9, 12, 18, 24 and $48 \mathrm{~h}$ of LPS injection. Within 6-24 h of LPS injection, the food intake and appetite of $M$. amblycephala decreased significantly, whereas the mRNA expression of leptin and mTOR increased significantly. Our results indicate that inflammatory cytokines may be the cause of appetite suppression in $M$. amblycephala fed a HFD
\end{abstract}

Key words: Megalobrama amblycephala: Appetite: High-fat diets: Inflammatory factors: Food intake

Recent evidence from studies in humans ${ }^{(1)}$ and rats $^{(2,3)}$ indicated that high-fat diet (HFD) intake was associated with appetite suppression and reduced sensitivity to the current food value. Fish was not an exception. Studies had reported that grass carp (Ctenopharyngodon idella) had been adopted as a strategy to deal with exposure to high fat by reducing appetite $^{(4)}$. The use of fat-rich feeds is the current trend in intensive aquaculture for the protein-sparing and growth-promoting effects of such diets ${ }^{(5,6)}$. However, the suppression of appetite caused by excessive fat in diet would lead to a poor growth, which had limited the use of HFD in aquatic animals ${ }^{(4)}$. Therefore, it is meaningful to investigate the mechanism by which HFD induce appetite suppression.

As lipid is one of major nutrients in fish for supporting numerous physiological processes ${ }^{(7,8)}$, it is not surprising that lipid levels of diet were related to appetite in fish $^{(9)}$. The brain tissue of fish acts as an integrator of signals of relevance to energy balance and regulates appetite of fish by neuroendocrine secretion. Cocaine- and amphetamine- regulated transcript (CART) and neuropeptide Y (NPY) are two neuropeptides that mutually interact to inhibit or stimulate appetite $^{(10,11)}$. In teleost fish, evidence obtained in recent years pointed to the presence of sensor systems for changes in the levels of fatty acids (FA), which were related to the control of appetite through changes in mRNA expression of orexigenic and anorexigenic neuropeptides ${ }^{(12,13)}$. One of these mechanisms was based on FA metabolism through inhibition of carnitine palmitoyltransferase 1 (CPT1) to import fatty-acid-CoA into the mitochondria for oxidation ${ }^{(14-16)}$. Another possible element dominating FA-sensing systems was FA transport, in which FA bind to fatty acid translocase (FAT)/cluster of differentiation 36 (CD36) and further modulates transcription factors, such as sterol regulatory element-binding protein type 1c (SREBP1c) ${ }^{(13,17)}$. The activation of these systems was associated with the inhibition of the orexigenic NPY and the enhancement of the anorexigenic CART, ultimately leading to the decrease in food intake ${ }^{(18)}$. Several studies had focused on FA sensing systems that were connected to the regulation of appetite in fish; however, it was

Abbreviations: AKT, protein kinase B; CART, cocaine- and amphetamine-regulated transcript; CPT1, carnitine palmitoyltransferase 1; FA, fatty acid; FAT, fatty acid translocase; HFD, high-fat diet; I $\kappa$ B, inhibitor of $\kappa \mathrm{B}$; LPS, lipopolysaccharide; mTOR, mammalian target of rapamycin; NFD, normal-fat diet; NPY, neuropeptide Y; SREBP1c, sterol regulatory element-binding protein type 1c.

* Corresponding author: W.-B. Liu, email wbliu@njau.edu.cn 
still difficult to establish which factors acted in long-term experiment, as most of the available data originated from shortterm acute experiments ${ }^{(19)}$. In fact, the sensing systems characterised in rainbow trout whose activation in response to increased levels of oleate had been found to result in decreased food intake did not respond in the same way when fish were fed for 4 weeks with a lipid-enriched $\operatorname{diet}^{(20)}$. Was this also true for Megalobrama amblycephala? If so, which factors lead to weaker appetite? In this study, we will focus on a precise mechanism by which a HFD induces the suppressed appetite after long-term feeding of a HFD.

In addition to sensor systems for FA, the inflammatory factors are another potential contributor to the food intake of fish fed with HFD. In mammals, appetite suppression was a common and debilitating symptom that occurs as a consequence of acute and chronic inflammation ${ }^{(21)}$. In acute experimental models, administration of lipopolysaccharide (LPS), TNF- $\alpha$ and IL-6 directly reduced food intake by affecting a central system that integrated the action of numerous neuropeptides ${ }^{(22-24)}$. Moreover, chronic administration of LPS could induce feeding reduction in a dose-dependent manner ${ }^{(25)}$. Studies in mice had shown that the appetite suppression was caused by inflammatory factors, which were related to the activation of mammalian target of rapamycin (mTOR), and inhibition of mTOR could relieve inflammation-induced appetite suppression ${ }^{(26)}$. Studies in mammals have suggested that appetite suppression induced by chronic inflammation might be related to higher levels of leptin in the blood ${ }^{(27)}$. In mammals, leptin had a dual role as a hormone and a cytokine. As a hormone, it played a key function of modulating energy homoeostasis through mechanisms that included appetite ${ }^{(28)}$. One of the classic pathways by which leptin suppressed appetite was activation of mTOR phosphorylated by protein kinase $\mathrm{B}(\mathrm{Akt})^{(29)}$. As a cytokine, leptin had pro-inflammatory properties, and multiple inflammatory cytokines including TNF- $\alpha$ and IL- 6 stimulated up-regulation of leptin gene expression ${ }^{(30,31)}$. In fish, leptin played a key role in the regulation of food intake by altering orexigenic (such as NPY) and anorexigenic (such as CART) neuropeptides to regulate appetite ${ }^{(32)}$. In addition, a study in rainbow trout showed that leptin could also be activated by the AKT signalling pathway, as found in mammals ${ }^{(33)}$. All of these facts suggest that inflammatory factors were important in regulating appetite. However, there are no available studies in fish assessing the response of appetite to inflammation, as demonstrated in mammals. A recent article showed that feeding zebrafish (Danio rerio) with a HFD for 8 weeks caused an increase of inflammatory markers ${ }^{(34)}$. Our previous study also found that the expression of TNF- $\alpha$ in the liver of $M$. amblycephala fed HFD was significantly increased, suggesting that a chronic inflammation might be developed in the HFD group ${ }^{(35)}$. However, whether the low-chronic inflammation caused by high fat in fish was associated with food intake remained unknown.

Studies in fish have shown that long-term administration of HFD would lead to a reduction in food intake, but the precise mechanism still remained unknown. In this study, we aim to figure out the mechanism by which a HFD lead to weakened appetite.

\section{Methods}

\section{Ethics statement}

The care and use of animals and all experimental procedures involving animals were approved by the ethics committee, and conform to the 'ARRIVE' Guidelines for Reporting Animal Research $^{(36)}$.

\section{Experimental diets}

The composition of the basal diet and HFD is shown in Table 1. Fish meal, soyabean meal, cottonseed meal and rapeseed meal served as protein sources. Soyabean oil and fish oil were used as lipid sources. Wheat flour was adopted as a carbohydrate source. Dietary ingredients were ground into fine powder, thoroughly mixed and then blended with an additional $100 \mathrm{ml}$ of water $/ \mathrm{kg}$ of diet to form a soft dough, which was pelleted (without injected steam) using a Pillet Mill (Guangyuan Engineering Co., Ltd) with a 2 -mm-diameter die. The experiment feed was dried at air temperature at $28^{\circ} \mathrm{C}$ overnight and stored in sealed plastic bags at $-4^{\circ} \mathrm{C}$ until use. The proximate composition of the experimental diets was determined according to the standard Association of Official Analytical Chemists (AOAC) methodology.

Experimental diets were analysed for FA profiles using GC (6890 series; Agilent Technologies) according to the American Oil Chemists' Society method. The FA composition of the basal diet and HFD is shown in Table 2.

\section{Fish and experimental design}

Juvenile $M$. amblycephala were obtained from a local fish hatchery (Nanjing, China). Before the experiment, fish were reared

Table 1. Formulation and proximate composition of the experimental diets

\begin{tabular}{|c|c|c|}
\hline Ingredients (\%) & High-fat diet (\%) & Normal-fat diet (\%) \\
\hline Fish meal & 5 & 5 \\
\hline Soyabean meal & 30 & 30 \\
\hline Rapeseed meal & 15 & 15 \\
\hline Cottonseed meal & $10 \cdot 60$ & $8 \cdot 12$ \\
\hline Soyabean oil & 4.74 & $2 \cdot 17$ \\
\hline Fish oil & 4.74 & $2 \cdot 17$ \\
\hline Wheat bran & 3.98 & 7.65 \\
\hline Wheat flour & $22 \cdot 04$ & $26 \cdot 00$ \\
\hline $\mathrm{Ca}\left(\mathrm{H}_{2} \mathrm{PO}_{4}\right)_{2}$ & $1 \cdot 8$ & $1 \cdot 8$ \\
\hline Salt & 0.4 & 0.4 \\
\hline Ethoxyquinoline & 0.5 & 0.5 \\
\hline Premix $^{*}$ & 1.2 & 1.2 \\
\hline \multicolumn{3}{|c|}{ Proximate composition } \\
\hline Moisture & $12 \cdot 1$ & $11 \cdot 1$ \\
\hline Crude protein & 31.4 & $30 \cdot 8$ \\
\hline Crude lipid & $10 \cdot 3$ & $5 \cdot 1$ \\
\hline Energy (MJ/kg) & $19 \cdot 7$ & $17 \cdot 4$ \\
\hline
\end{tabular}


Table 2. Fatty acid composition of the normal-fat diet (NFD) and high-fat diet (HFD)

\begin{tabular}{lrr}
\hline & \multicolumn{2}{c}{ Diet } \\
\cline { 2 - 3 } Fatty acid & NFD & HFD \\
\hline C14:0 & $2 \cdot 74$ & $2 \cdot 85$ \\
C15:0 & 0.19 & $0 \cdot 16$ \\
C16:0 & 18.54 & $16 \cdot 34$ \\
C17:0 & 0.13 & 0.25 \\
C18:0 & 4.76 & $4 \cdot 19$ \\
C20:0 & 0.45 & 0.37 \\
C16:1 & 0.78 & 0.94 \\
C18:1 & 28.28 & 35.59 \\
C20:1 & 1.29 & 0.98 \\
C22: & 1.72 & 1.22 \\
C18:2 & 18.45 & 19.12 \\
C20:2 & 0.98 & 0.30 \\
C18:3 & 2.78 & 1.95 \\
C20:3 & 0.07 & 0.04 \\
C22:3 & 0.06 & 0.05 \\
C20:4 & 0.14 & 0.87 \\
C22:4 & 0.08 & 0.07 \\
C20:5 & 0.04 & 0.37 \\
C22:5 & 0.19 & 0.16 \\
C22:6 & 1.06 & 1.47 \\
\hline
\end{tabular}

in several floating net cages $(2 \times 1 \times 1 \mathrm{~m}$, length:width:height $)$ for 1 month to acclimate to the experimental conditions. During this period, fish were domesticated to eat in the upper aquifer. After a 1-month acclimation, 120 fish of similar sizes (average initial weight: 40.00 (SEM 0.35) g) were randomly distributed into eight cages, which were anchored in an outdoor pond. Juvenile $M$. amblycephala were divided into two groups for $60 \mathrm{~d}$ and each treatment had four replicates. Fish in the control group were fed a normal-fat diet (NFD, 6\% lipid), whereas those in the treatment group were fed a HFD ( $11 \%$ lipid). Body weight of fish in each replicate was measured once every $15 \mathrm{~d}$, and food intake was determined every $3 \mathrm{~d}$. We kept feeding until the fish did not eat in the upper layer. Fish were hand-fed to apparent satiation three times daily (08.00, 12.00 and 17.00 hours) for $60 \mathrm{~d}$. Fish intake was indicated by cumulative food intake per fish. Cumulative food intake per fish $=$ cumulative food intake in each cage/the number of fish in each cage. Water temperature varied from 27 to $30^{\circ} \mathrm{C}$, and $\mathrm{pH}$ fluctuated between $7 \cdot 2$ and $7 \cdot 6$. Ammonia nitrogen and nitrite nitrogen were maintained below 0.4 and $0.064 \mathrm{mg} / \mathrm{l}$, respectively. Dissolved oxygen was maintained above $6.00 \mathrm{mg} / \mathrm{l}$ during the feeding trial.

\section{Sample collection}

At the end of the feeding trial, approximately $24 \mathrm{~h}$ after the last feeding, sampling was started. All fish were quickly removed from each cage and anaesthetised in diluted MS-222 (tricaine methanesulfonate; Sigma) at a concentration of $100 \mathrm{mg} / \mathrm{l}$. Total number and weight of fish in each cage were determined to calculate the growth performance. A total of six fish were randomly removed from each cage and blood sample was collected by caudal vein puncture using heparinised syringes coated with lithium heparin as an anti-coagulant. After centrifugation $\left(3000 \mathrm{~g}\right.$ for $10 \mathrm{~min}$ at $4^{\circ} \mathrm{C}$ ), plasma was stored at $-80^{\circ} \mathrm{C}$ for subsequent analysis. Then, fish were killed immediately by decapitation, individual brains were dissected over an ice bed, washed thoroughly with chilled saline $(0.89 \mathrm{~g} / \mathrm{l} \mathrm{NaCl})$ and stored at $-80^{\circ} \mathrm{C}$ for the analysis of gene expression.

\section{Plasma biochemical assays}

The serum level of total TAG was measured with a Hitachi 7180 800T/H automatic biochemistry analyzer (Hitachi). Measurement of NEFA concentration was carried out in duplicate using an enzymatic colourimetric method, using the Wako NEFA C kit (Wako Chemicals Inc.). The concentration of serum leptin was determined using the mouse insulin ELISA kit according to the manufacturer's instructions (R\&D Systems, no. DLP00).

\section{Plasma inflammatory markers}

The concentrations of serum TNF- $\alpha$, IL- 6 and monocyte chemoattractant protein-1 (MCP-1) were measured using cytokinespecific ELISA kits according to the manufacturer's instructions (R\&D Systems, nos MTA00B, D6050 and DCP00). Plasma nitric oxide (NO) levels were determined by the nitrate reductase assay using a commercial kit (reference no. A012) produced by Jianchen, Bioengineering Institute (Nanjing, China).

\section{Quantitative RT-PCR analysis}

Total RNA was isolated from liver and brain using Trizol reagent (Invitrogen) according to the manufacturer's instructions, and treated with RQ1 RNase-free DNase (Promega) to eliminate genomic DNA contamination. Quantity and purity of isolated RNA were later determined by absorbance measures at 260 and $280 \mathrm{~nm}$, respectively. The integrity of RNA was tested by electrophoresis in $1.0 \%$ formaldehyde denaturing agarose gels. The mRNA expressions were detected in brain and adipose tissue.

Total RNA was reverse-transcribed using a RT-PCR Kit (Takara) according to the instructions of SYBR ${ }^{\circledR}$ PrimeScript $^{\mathrm{TM}}$. The volume of PCR reactions was $10 \mu \mathrm{l}$, containing $0.5 \mu \mathrm{l}$ of deoxy-ribonucleoside triphosphate (dNTP) mixture $(10 \mathrm{~mm}$ each), $0 \cdot 25 \mu \mathrm{l}$ of RNase inhibitor $(40 \mathrm{U} / \mu \mathrm{l}), 0.5 \mu \mathrm{l}$ of primer $(50 \mathrm{~mm}), 0 \cdot 25 \mu \mathrm{l}$ of ExScript ${ }^{\mathrm{TM}}$ RTase $(200 \mathrm{U} / \mu \mathrm{l}), 2 \cdot 0 \mu \mathrm{l}$ of buffer $(5 \times)$ and $6.5 \mu \mathrm{l}$ of diethyl pyrocarbonate (DEPC) water. The cycling conditions were set as follows: $42^{\circ} \mathrm{C}$ for $40 \mathrm{~min}, 90^{\circ} \mathrm{C}$ for $2 \mathrm{~min}$ and $4^{\circ} \mathrm{C}$ thereafter.

After reverse transcription, target complementary DNA (CDNA) was diluted and amplified by RT-PCR using an SYBR Green II Fluorescence Kit (Takara Bio Inc.) to determine the mRNA abundance. Primers were designed based on the available sequences in $M$. amblycephala (Table 3). RT-PCR was conducted in a MiniOpticon real-time detector (Bio-Rad). The protocol consisted of forty cycles, with the first step of denaturation at $95^{\circ} \mathrm{C}$ for $30 \mathrm{~s}$, a final extension of $95^{\circ} \mathrm{C}$ for $5 \mathrm{~s}$ and annealing at $60^{\circ} \mathrm{C}$ for $34 \mathrm{~s}$. Each sample was run in triplicate, and PCR reactions without target CDNA were adopted as negative controls. At the end of the reaction, the fluorescent data were converted into $C_{\mathrm{t}}$ values. Then, each transcript level was normalised to $\beta$-actin using the $2^{-\Delta \Delta} C_{\mathrm{t}}$ method. 
Table 3. Real-time PCR primer sequences

\begin{tabular}{|c|c|c|}
\hline Gene & Sequences of primers & GenBank no. \\
\hline$N P Y$ & $\begin{array}{l}\text { GAGACCCACCGAGCAAGAA } \\
\text { AGGCGAACAAGAGGAAGGC }\end{array}$ & JQ301475.1 \\
\hline$C A R T$ & $\begin{array}{l}\text { GGACCCGAATCTGACAAACGA } \\
\text { TTTGCCGATTCTTGACCCTTT }\end{array}$ & AM498379.1 \\
\hline$T N F-a$ & $\begin{array}{l}\text { TCAAAGTCAGGCGTATGG } \\
\text { CTGGCTGTAGACGAAGTAAAT }\end{array}$ & KP192129.1 \\
\hline IL-6 & $\begin{array}{l}\text { GTCCTGACGTGGTACAAAGA } \\
\text { CGTTTGGTCTCGTGTTTGAT }\end{array}$ & KJ755058.1 \\
\hline$N F-K B$ & $\begin{array}{l}\text { GAAGAAGGATGTGGGAGATGC } \\
\text { TGTTGTCGTAGATGGGCTGAG }\end{array}$ & KJ526214 \\
\hline $\operatorname{lk} B$ & $\begin{array}{l}\text { TCTTGCCATTATTCACGAGG } \\
\text { TGTTACCACAGTCATCCACCA }\end{array}$ & KJ125069 \\
\hline PPARa & $\begin{array}{l}\text { GTGCCAATACTGTCGCTTTCAG } \\
\text { CCGCCTTTAACCTCAGCTTCT }\end{array}$ & HM140628 \\
\hline CPT1 & $\begin{array}{l}\text { TACTTCCAAAGCGGTGAG } \\
\text { AGAGGTATTGTCCGAGCC }\end{array}$ & KJ141198.1 \\
\hline$A C O$ & $\begin{array}{l}\text { GCTCAACCCTGGCATACT } \\
\text { CTGGCTCAGCTTTACACG }\end{array}$ & Xu et al. ${ }^{(50)}$ \\
\hline FAT/CD36 & $\begin{array}{l}\text { CTTCTTCCTGGTGGCATCCT } \\
\text { TGGGTTCAACAGTCGCATTCT }\end{array}$ & $\mathrm{Xu}$ et al. ${ }^{(50)}$ \\
\hline SREBP1C & $\begin{array}{l}\text { GCTGGCGTGTCGCTATCT } \\
\text { TGTTGGCAGTCGTGGAGG }\end{array}$ & Xu et al. ${ }^{(50)}$ \\
\hline Leptin & $\begin{array}{l}\text { GGGAGATTCCTGCTTGAGT } \\
\text { AAGGCTGCTTCTTCTTTGT }\end{array}$ & KJ193854.1 \\
\hline$A K T$ & $\begin{array}{l}\text { GCTGGGTAAAGGCACGTTTG } \\
\text { CTCTCGGTGACCGTATGAGC }\end{array}$ & Pan et al. ${ }^{(51)}$ \\
\hline mTOR & $\begin{array}{l}\text { TTTACACGAGCAAGTCTACGGA } \\
\text { CTTCATCTTGGCTCAGCTCTCT }\end{array}$ & Xu et al. ${ }^{(52)}$ \\
\hline EF1a & $\begin{array}{l}\text { CTTCTCAGGCTGACTGTGC } \\
\text { CCGCTAGCATTACCCTCC }\end{array}$ & X77689.1 \\
\hline
\end{tabular}

$N P Y$, neuropeptide Y; $C A R T$, cocaine- and amphetamine-regulated transcript; IKB inhibitor of $K \mathrm{~B}$; $C P T 1$, carnitine palmitoyltransferase 1; $A C O$, acetyl-CoA oxidase; $F A T$, fatty acid translocase; SREBP1c, sterol regulatory element-binding protein type 1c; $A K T$, protein kinase $\mathrm{B}$; $m T O R$, mammalian target of rapamycin; EF1a, elongation factor $1 a$.

\section{Lipopolysaccharide injection}

Megalobrama amblycephala juveniles were obtained from the local fish hatchery (Nanjing, China). Before the experiment, fish were kept in tanks to acclimate to the experiment by feeding with a commercial diet, which contains $6 \%$ lipid and $30 \%$ crude protein, for 2 weeks. Next, 160 fish with a mean body weight of 40.00 ( SEм 0.5) g were randomly distributed into sixteen tanks (300 litres each). Fish were divided into two treatment groups and each treatment had eight replicates: one group was injected intraperitoneally with LPS (injected intraperitoneally with LPS) at dosages of $2 \mathrm{mg} / \mathrm{kg}$ and another group was injected with PBS as the control group. Fish were allowed to access food at 3, 6, 9, 12, 18, 24 and $48 \mathrm{~h}$ and food intake was recorded. In all, two groups with four replicates were used for brain tissue sampling, for gene analysis. Other two groups with four replicates were used for determination of food intake.

\section{Statistical analysis}

Data concerning chronic experiment were subjected to independent sample $t$ test using the SPSS program version 19.0 (SPSS Inc.) for Windows, after testing the normality and homogeneity of variances with the Levene test. Data for LPS injection were subjected to one-way ANOVA and independent sample $t$ test using the SPSS program version 19.0 for Windows. The normality and homogeneity were tested using Levene's test

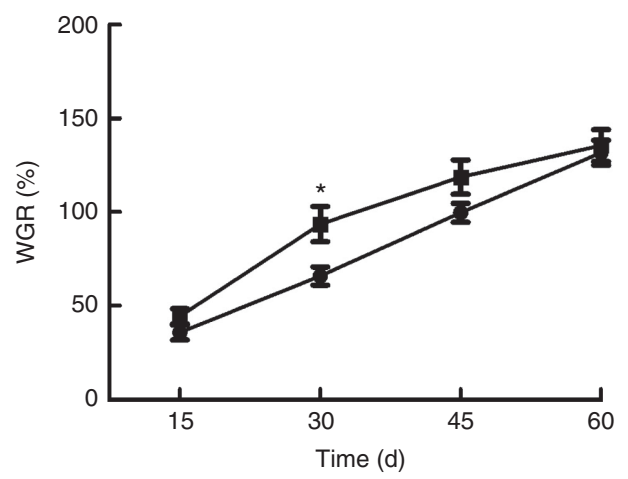

Fig. 1. Weight gain rate (WGR) in blunt snout bream (Megalobrama amblycephala) fed a normal-fat diet (NFD, - - ) or high-fat diet (HFD, - -) for $60 \mathrm{~d}$. WGR $=$ (final body weight - initial body weight $) \times 100 /$ initial body weight. Values are means $(n 4)$, with their standard errors represented by vertical bars. * Mean values were significantly different between treatment and control groups $(P<0.05)$.

for equality of variance. Duncan's multiple-range test was used to compare the significance of gene expression and food intake among different times after injection of PBS and LPS. Independent sample $t$ test was used to compare gene expression and food intake between the fish injected with PBS and LPS at the same times. Differences were considered statistically significant at $P<0 \cdot 05$. All data were presented as means with their standard error of the mean of four replicates.

\section{Results}

\section{Growth performance}

Growth performance of $M$. amblycephala fed different dietary fat levels is presented in Fig. 1. Weight gain rate (WGR) was significantly $(P<0.05)$ higher in the HFD for $30 \mathrm{~d}$ than that in the NFD, whereas WGR showed no significant differences between two groups fed for $60 \mathrm{~d}$. Food intake of $M$. amblycephala fed different dietary fat levels is presented in Fig. 2. Food intake of M. amblycephala tended to decline significantly when fish were fed the HFD for $48 \mathrm{~d}$.

\section{Plasma biochemistry parameters}

Plasma leptin, TAG and NEFA concentrations were reported in Table 4. Compared with the basic control group (NFD), plasma leptin, TAG and NEFA concentrations were significantly increased in the group fed the HFD $(P<0.05)$.

\section{Inflammatory markers}

Data on inflammatory marker concentrations in plasma are shown in Table 5. Compared with the basic control group (NFD), the HFD significantly increased $(P<0.05)$ the circulating protein levels of pro-inflammatory cytokines TNF- $\alpha$, IL- 6 and MCP-1. Similarly, the concentration of NO, a marker of inflammation, was significantly higher $(P<0.05)$ than that of the control group (NFD). 


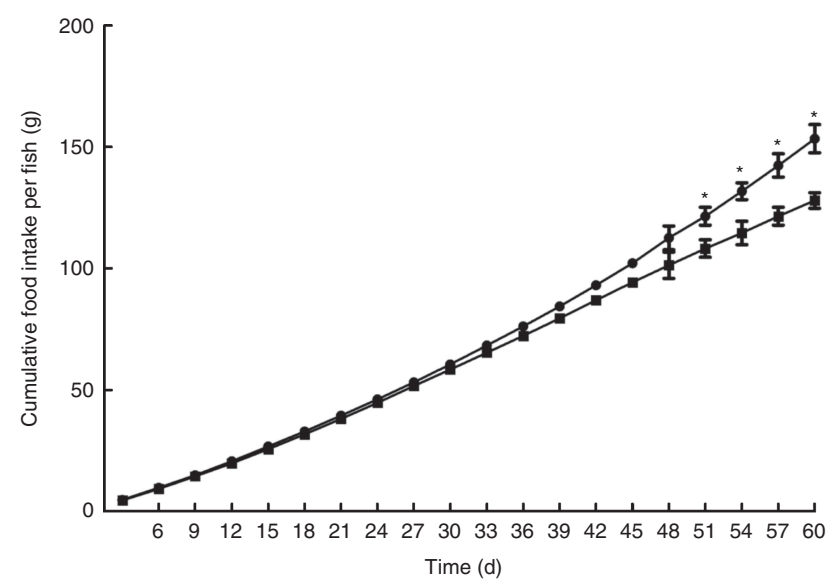

Fig. 2. Cumulative food intake in blunt snout bream (Megalobrama amblycephala) fed a normal-fat diet (NFD, - - ) or high-fat diet (HFD, -) for $60 \mathrm{~d}$. Values are means $(n 4)$, with their standard errors represented by vertical bars. * Mean values were significantly different between treatment and control groups $(P<0.05)$.

Table 4. Plasma biochemistry parameters in Megalobrama amblycephala fed the normal-fat diet (NFD) or high-fat diet (HFD) for $60 \mathrm{~d}^{*}$

\begin{tabular}{|c|c|c|c|c|}
\hline & \multicolumn{2}{|c|}{ Diet } & \multirow[b]{2}{*}{ SEM } & \multirow[b]{2}{*}{$P$} \\
\hline & NFD & HFD & & \\
\hline TAG (mmol/l) & 1.67 & $3 \cdot 24$ & 0.32 & 0.001 \\
\hline NEFA $(\mathrm{mmol} / \mathrm{l})$ & 0.33 & 0.69 & 0.07 & 0.002 \\
\hline Leptin (ng/ml) & 1.51 & $2 \cdot 17$ & 0.16 & 0.022 \\
\hline
\end{tabular}

* Four fish per group for data analysis ( $n 4)$.

Table 5. Inflammatory markers in Megalobrama amblycephala fed the normal-fat diet (NFD) or high-fat diet (HFD) for $60 \mathrm{~d}^{*}$

\begin{tabular}{|c|c|c|c|c|}
\hline & \multicolumn{2}{|c|}{ Diet } & \multirow[b]{2}{*}{ SEM } & \multirow[b]{2}{*}{$P$} \\
\hline & NFD & HFD & & \\
\hline TNF- $a(\mathrm{ng} / \mathrm{ml})$ & $18 \cdot 15$ & $30 \cdot 84$ & $2 \cdot 85$ & 0.013 \\
\hline IL-6 (ng/ml) & 21.41 & 39.86 & $4 \cdot 61$ & 0.016 \\
\hline MCP-1 (ng/ml) & 48.02 & $65 \cdot 80$ & 3.93 & 0.007 \\
\hline $\mathrm{NO}(\mu \mathrm{mol} / \mathrm{l})$ & 9.19 & $16 \cdot 10$ & $1 \cdot 71$ & 0.028 \\
\hline
\end{tabular}

MCP-1, monocyte chemoattractant protein-1; NO, nitric oxide.

${ }^{*}$ Four fish per group for data analysis $(n 4)$.

\section{Expressions of genes involved in appetite, fatty acid sensing and inflammation}

The expressions of appetite genes in $M$. amblycephala fed different dietary fat levels are presented in Fig. 3. Compared with the basic control group (NFD), the expression of NPY was significantly lower and the expression of CART was significantly higher in the HFD group $(P<0.05)$.

The expressions of genes involved in FA sensing in M. amblycephala fed different dietary fat levels are presented in Fig. 4. The mRNA levels of PPAR $\alpha, C P T 1$ and acetyl-CoA oxidase $(A C O)$ showed no significant difference $(P>0.05)$ between the two groups, whereas the mRNA levels of FAT/ $C D 36$ and $S R E B P 1 C$ were higher in the HFD group than those in the NFD group $(P<0.05)$.

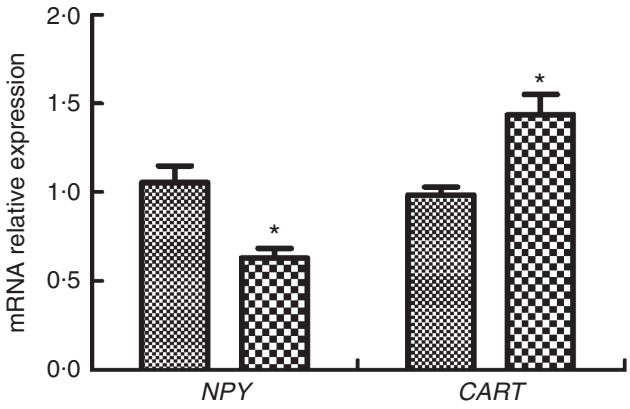

Fig. 3. Relative gene expression of neuropeptide $Y(N P Y)$ and cocaine- and amphetamine-regulated transcript $(C A R T)$ in brain of blunt snout bream (Megalobrama amblycephala) fed a normal-fat diet (NFD, :) or high-fat diet (HFD, 9) for $60 \mathrm{~d}$. Values are means $(n 4)$, with their standard errors represented by vertical bars. * Mean values were significantly different between treatment and control groups $(P<0.05)$.

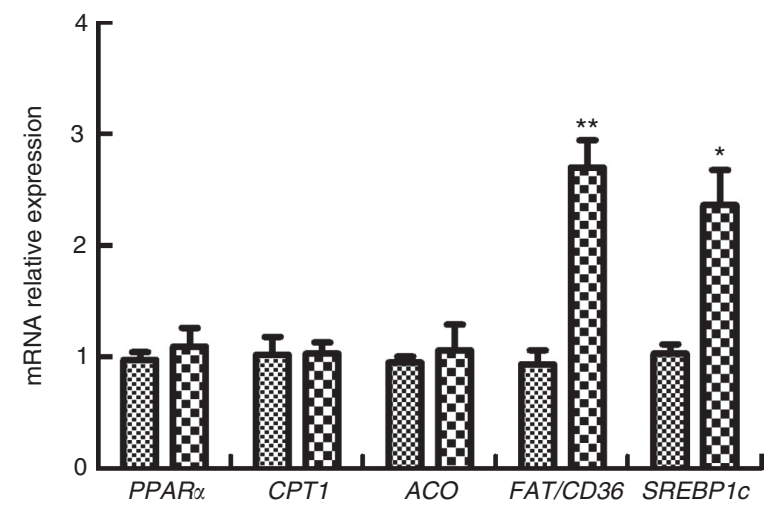

Fig. 4. Relative gene expression of $P P A R a$, carnitine palmitoyltransferase 1 (CPT1), acetyl-CoA oxidase $(A C O)$, fatty acid translocase (FAT/CD36) and sterol regulatory element-binding protein type 1c (SREBP1C) in brain of blunt snout bream (Megalobrama amblycephala) fed a normal-fat diet (NFD, \&) or high-fat diet (HFD, $\mathbf{B}$ ) for $60 \mathrm{~d}$. Values are means $(n 4)$, with their standard errors represented by vertical bars. Mean values were significantly different between treatment and control groups: ${ }^{\star} P<0.05$ and ${ }^{\star *} P<0.01$.

The expressions of genes involved in inflammatory factors in M. amblycephala fed different dietary fat levels are presented in Fig. 5. Compared with the basic control group (D1), the mRNA levels of $I L-6, T N F-\alpha$ and $N F-\kappa B$ were significantly increased $(P<0.05)$ in fish of the HFD group, and the mRNA level of inhibitor of $\kappa \mathrm{B}(I \kappa B)$ showed an opposite trend. Furthermore, genes involved in cellular signalling, $m T O R, A k t$ and leptin were significantly increased $(P<0.05)$ in the HFD group.

\section{Effect of lipopolysaccharide injection}

As shown in Figs. 6 and 7, the change of food intake among different times after PBS injection shared similar trends with those in the LPS-injection group. Food intake significantly reduced $(P<0.05)$ at $6 \mathrm{~h}$ after PBS and LPS injection, reached the lowest at $18 \mathrm{~h}(P<0.05)$ and rose back to normal at $48 \mathrm{~h}$. Comparing food intake between the fish injected with PBS and LPS at the same times, at 6, 9, 12, 18 and $24 \mathrm{~h}$, the food intake in fish injected with LPS was significantly lower $(P<0.05)$ than those of PBS injection. The expression patterns of $N P Y$ shared similar trend with the profiles of food intake (Fig. 7(A)). In terms 


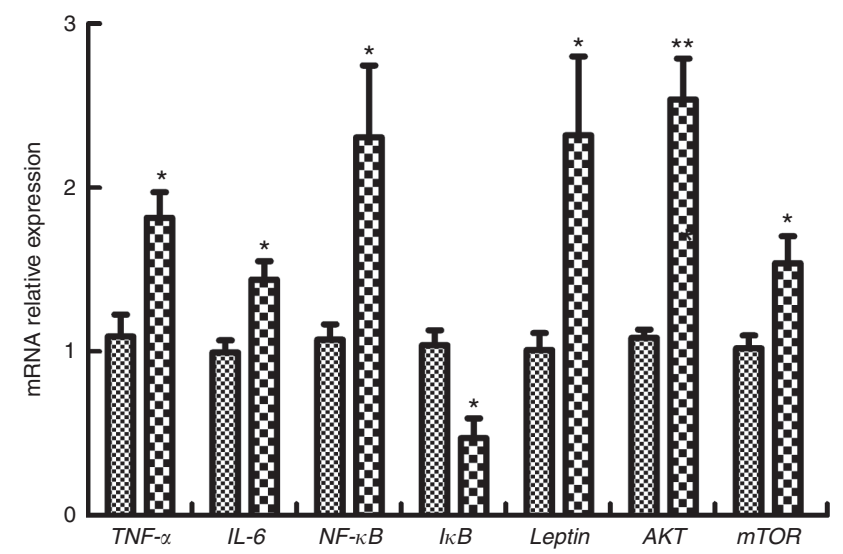

Fig. 5. Relative gene expression of $T N F-a, I L-6, N F-K B$, inhibitor of $\kappa B(I K B)$, leptin, mammalian target of rapamycin $(m T O R)$ and protein kinase $\mathrm{B}(A K T)$ in brain of blunt snout bream (Megalobrama amblycephala) fed a normal-fat diet (NFD, : $)$ or high-fat diet (HFD, Q 8 ) for $60 \mathrm{~d}$. Values are means $(n 4)$, with their standard errors represented by vertical bars. Mean values were significantly different between treatment and control groups: ${ }^{\star} P<0.05$, ${ }^{* *} P<0.01$.

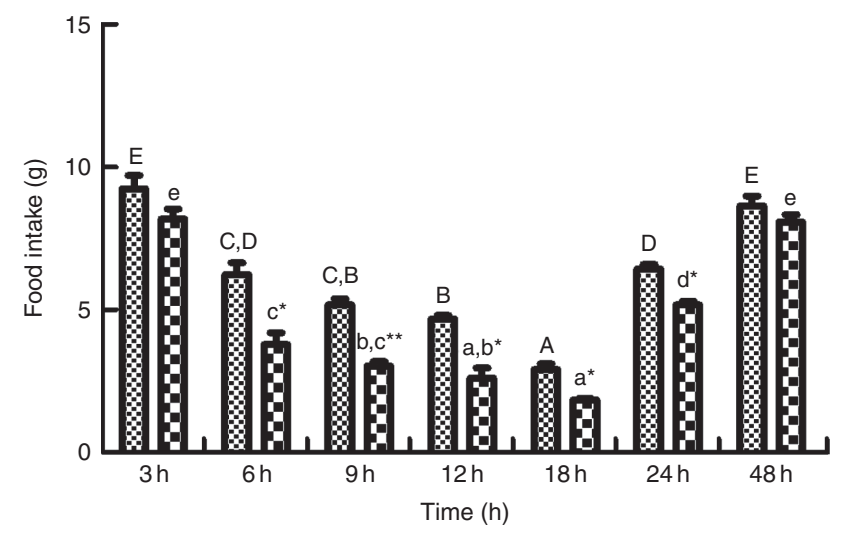

Fig. 6. The food intake of blunt snout bream injected intraperitoneally with PBS (圆) and lipopolysaccharide (LPS (B), $2 \mathrm{mg} / \mathrm{kg}$ ). Values are means $(n 4)$, with their standard errors represented by vertical bars. $A, B, C, D, E$ Mean values with unlike letters were significantly different in the same model among groups at 3 , $6,9,12,18,24$ and $48 \mathrm{~h}$ after PBS was injected $(P<0.05))^{\mathrm{a}, \mathrm{b}, \mathrm{c}, \mathrm{d}, \mathrm{e}}$ Mean values with unlike letters were significantly different in the same model among groups at $3,6,9,12,18,24$ and $48 \mathrm{~h}$ after LPS was injected $(P<0.05)$. Meanwhile, the star bars also indicate the significance between the two treatments (PBS and LPS) at same time points. Mean values were significantly different between treatment and control groups: ${ }^{*} P<0.05$ and ${ }^{* *} P<0.01$.

of CART expression, an opposite trend with NPY was observed (Fig. 7(B)). CART transcripts were quickly up-regulated $(P<0.05)$ at $6 \mathrm{~h}$ after PBS and LPS injection, reached the peak level at $18 \mathrm{~h}(P<0.05)$ and dropped back to normal level at $48 \mathrm{~h}$. At 6, 9, 12, 18 and $24 \mathrm{~h}$, the mRNA levels of CART in fish injected with LPS were significantly higher $(P<0.05)$ than those of PBS.

The mRNA expression levels of TNF- $\alpha$, IL- 6 , leptin and mTOR are shown in Fig. 8(A)-(D). No significant differences in mRNA expression of TNF- $\alpha$ and IL- 6 were observed among different times after PBS injection. However, the mRNA levels of TNF- $\alpha$ were increased significantly $(P<0.05)$ and peaked at $6 \mathrm{~h}$ after LPS injection and descended down to normal level at $48 \mathrm{~h}$
(Fig. 8(A)). The expression of $I L-6$ increased $(P<0.05)$ and peaked at $6 \mathrm{~h}$ after LPS injection, increased $(P<0.05)$ again at $12 \mathrm{~h}$ after a down-regulation at $9 \mathrm{~h}$, followed by a recovery at $48 \mathrm{~h}$ (Fig. 8(B)). Comparing mRNA expressions of $T N F-\alpha$ and $I L-6$ between the fish injected with PBS and LPS at the same times, at 6, 9, 12, 18 and $24 \mathrm{~h}$, the expression level of $T N F-\alpha$ and $I L-6$ significantly up-regulated $(P<0.05)$ in fish injected with LPS than those of PBS (Fig. 8(A) and (B)). Leptin transcripts were significantly up-regulated $(P<0.05)$ at $6,9,12$ and $24 \mathrm{~h}$ after PBS injection, whereas leptin transcripts were significantly up-regulated $(P<0.05)$ and peaked at 6 after LPS injection, and then descended down to normal level at $48 \mathrm{~h}$. The mRNA expression of $m$ TOR was significantly $(P<0.05)$ elevated at $12 \mathrm{~h}$ after PBS injection, whereas $m T O R$ transcripts were significantly up-regulated $(P<0.05)$ at $6 \mathrm{~h}$ after LPS injection, reached the highest level at $9 \mathrm{~h}(P<0.05)$ and descended down to normal level at $48 \mathrm{~h}$. Comparing mRNA expressions of leptin and $m$ TOR between the fish injected with PBS and LPS at the same times, at 6, 9, 12 and $18 \mathrm{~h}$, the expression level of leptin and mTOR significantly up-regulated $(P<0.05)$ in fish injected with LPS than those of PBS.

\section{Discussion}

The use of fat-rich feeds was a current trend in intensive aquaculture for saving protein; however, studies in recent years have shown that excess fat in diet may cause a poor growth performance $^{(4)}$. Similar results were also observed from previous research in our laboratory - that HFD show no significant effects and tend to reduce the growth performance of M. amblycephala ${ }^{(37)}$. In this study, we found that the impact of a HFD on growth was time-dependent in $M$. amblycephala. Fish fed the HFD for $30 \mathrm{~d}$ showed a significant elevation in WGR, whereas there was no significant difference in WGR between two groups when fish were fed diets with different fat levels for $60 \mathrm{~d}$. We compared several experiments in rainbow trout and found similar results. Rainbow trout fed a lipid-enriched diet for 4 weeks exhibited a significant increase in $\mathrm{WRG}^{(20)}$, whereas trout fed a HFD for 7 weeks showed no significant differences in $\mathrm{WRG}^{(38)}$. Moreover, a recent study in zebrafish also showed similar results - that a sharply increasing trend of weight in zebrafish fed HFD was observed, but the increase of weight was slower after feeding HFD for 4 weeks ${ }^{(34)}$. The reason why a short-term $(30 \mathrm{~d})$ HFD could promote growth but a long-term (60 d) HFD failed might be associated with the appetite suppression of fish in the HFD group. In this study, fish fed a HFD for $60 \mathrm{~d}$ showed a decrease in food intake, whereas there was no significant difference in food intake during the first $30 \mathrm{~d}$. Other comparable studies carried out in rainbow trout similarly observed a significant decreased food intake owing to the higher dietary lipid content ${ }^{(9)}$, whereas trout fed a HFD for 4 weeks displayed no decrease in food intake ${ }^{(20)}$. Lower food intake observed in longterm feeding of HFD may be attributed to the slowness in growth performance of $M$. amblycephala. It is well known that food intake of fish was related to the expression of neuropeptides in the brain ${ }^{(39,40)}$. In the present study, $N P Y$ mRNA levels were significantly down-regulated, whereas the opposite trend was 

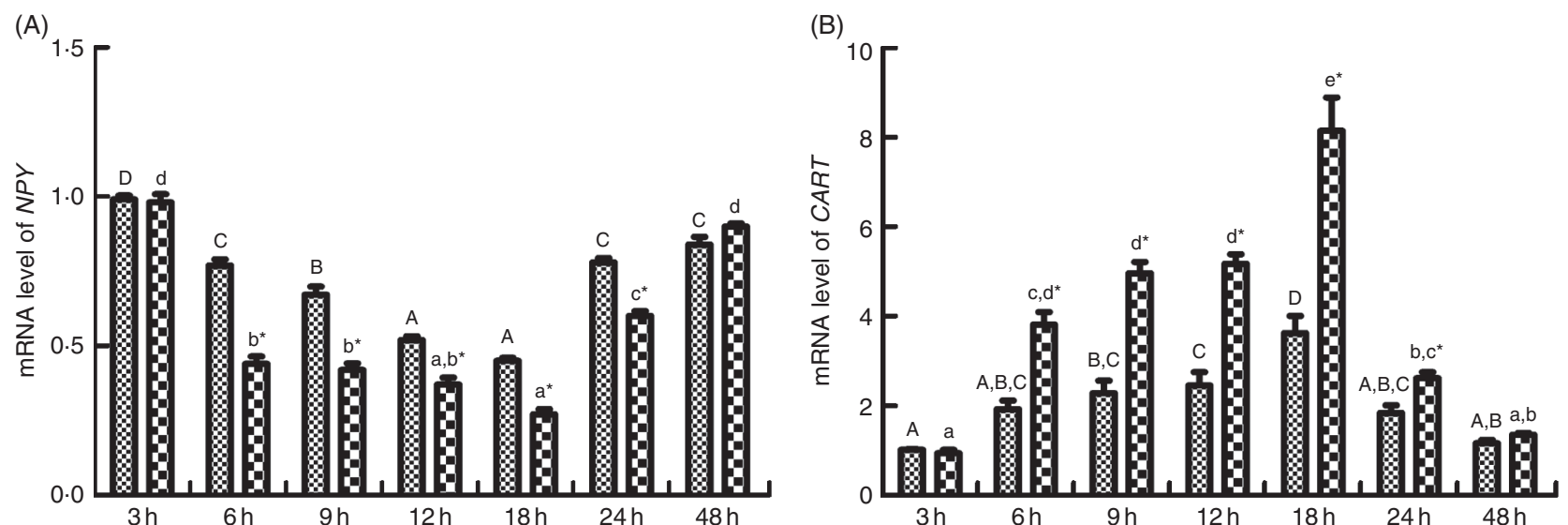

Fig. 7. Relative gene expression of neuropeptide $Y(N P Y, A)$ and cocaine- and amphetamine-regulated transcript (CART, B) in brain of blunt snout bream injected intraperitoneal with lipopolysaccharide (LPS (B), $2 \mathrm{mg} / \mathrm{kg}$ ) and PBS (圈). Values are means ( $n$ 4), with their standard errors represented by vertical bars. A,B,C,D Mean values with unlike letters were significantly different in the same model among groups at $3,6,9,12,18,24$ and $48 \mathrm{~h}$ after PBS was injected $(P<0.05)$. ${ }^{a, b, c, d}$ Mean values with unlike letters were significantly different in the same model among groups at 3, 6, 9, 12, 18, 24 and $48 \mathrm{~h}$ after LPS was injected ( $P<0.05)$. Meanwhile, the star bars also indicate the significance between the two treatments (PBS and LPS) at same time points. ${ }^{*}$ Mean values were significantly different between treatment and control groups $(P<0.05)$.
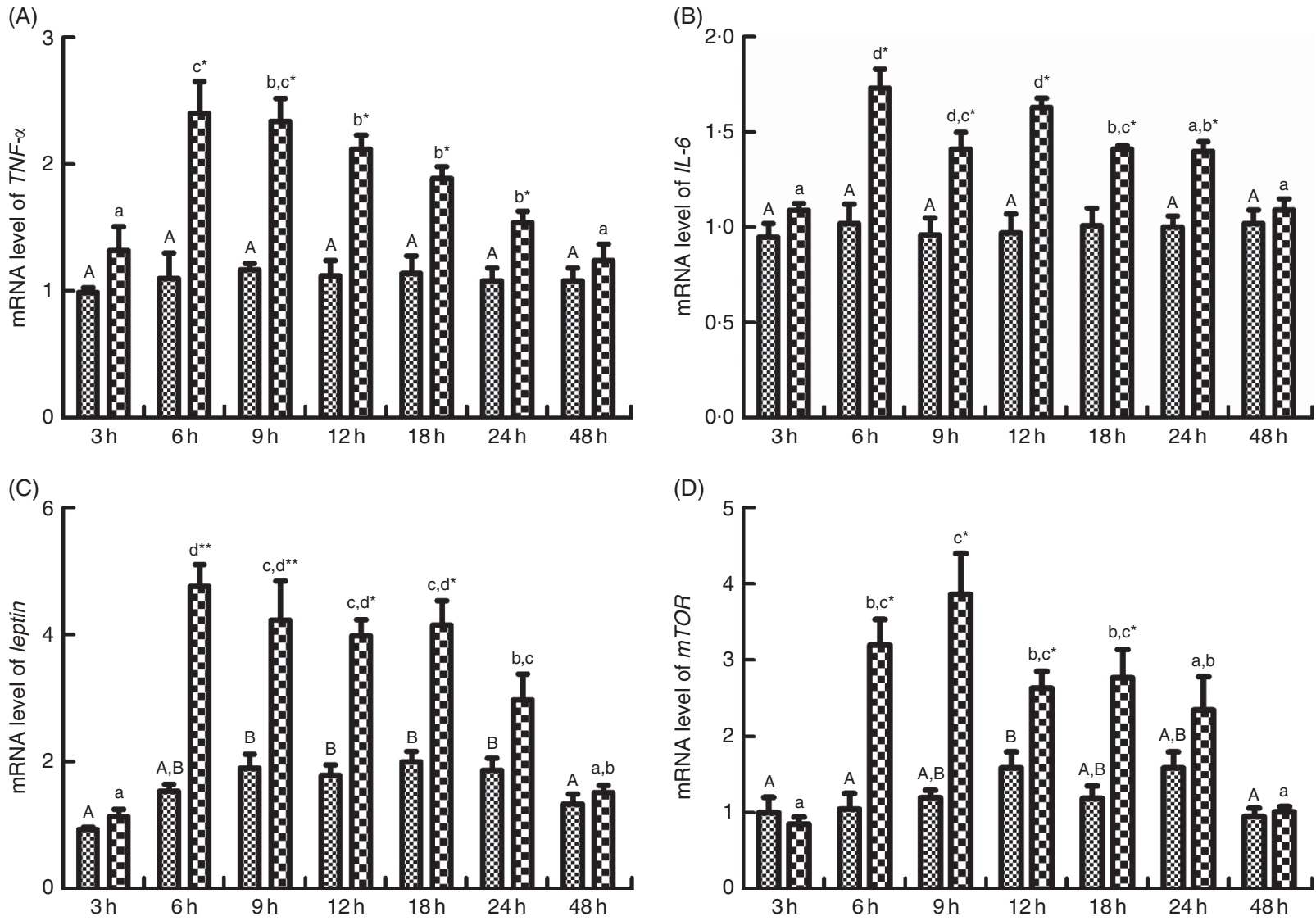

Fig. 8. Relative gene expression of $T N F-a(A), I L-6(B)$, leptin (C) and mammalian target of rapamycin ( $m T O R$, D) in brain of blunt snout bream injected intraperitoneally with PBS (圈) and lipopolysaccharide (LPS (B), $2 \mathrm{mg} / \mathrm{kg}$ ). Values are means $(n 4)$, with their standard errors represented by vertical bars. $\mathrm{A}, \mathrm{B}$ Mean values with unlike letters were significantly different in the same model among groups at $3,6,9,12,18,24$ and $48 \mathrm{~h}$ after PBS was injected $(P<0.05)$. ${ }^{\text {a,b,c,d }}$ Mean values with unlike letters were significantly different in the same model among groups at $3,6,9,12,18,24$ and $48 \mathrm{~h}$ after LPS was injected $(P<0.05)$. Meanwhile, the star bars also indicate the significance between the two treatments (PBS and LPS) at same time points. Mean values were significantly different between treatment and control groups: * $P<0.05$ and ${ }^{\star *} P<0.01$. 
observed in CART mRNA levels in the brain of fish offered a HFD, indicating that HFD could suppress appetite. Similar results in grass carp had also been reported: HFD feeding for 8 weeks contributed to elevated mRNA level of CART and decreased mRNA level of $N P Y$, actions both in favour of suppressed appetite $^{(4)}$. In teleost fish, evidence obtained in recent years was linked to the presence of sensor systems for FA, which were related to the control of appetite through changes in the expression of orexigenic neuropeptides ${ }^{(13,19)}$. In addition, suppression of appetite in mammals was a common and debilitating symptom that occured as a consequence of acute and chronic inflammation $^{(27)}$. In the current study, the circulating levels of FA and inflammatory factors in plasma were elevated significantly after long-term high-fat feeding. As both levels of FA and inflammatory factors were potential factors that induced appetite suppression, the next context would focus on exploring the precise mechanism of appetite suppression induced by high fat.

In teleost fish, short-term acute experiments, such as intraperitoneal or in vitro administration of oleate (long-chain fatty acids) or octanoate (medium-chain fatty acids), had demonstrated the presence of sensor systems for FA, which perceived the levels of FA contributing to changes in neuropeptide expression $^{(14,15)}$. However, the precise connection between changes in FA sensing and the expression of neuropeptide factors was not known even in mammals, although several possibilities had been suggested in fish, such as suppression of FA mitochondrial metabolism and activation of FA transport $^{(16,17,19)}$. In this experiment, the HFD significantly increased circulating FA level, which was evidenced by elevated concentrations of TAG and NEFA in plasma. However, whether this persistent hyperlipidaemia resulting from a long-term HFD was linked to appetite suppression had not been explored. It was well reported that PPAR $\alpha$ could facilitate the metabolism of FA by regulating the key enzymes involved in FA oxidation, such as CPT1, which was regarded as a reliable marker of mitochondrial FA oxidation $^{(41)}$. Data in current experiment showed that the abundance of PPAR $\alpha$ and CPT1 gene expression in brain was not affected by the fat level in diets. In addition, the gene expression of $A C O$, known as enzymes regulating FA oxidation, showed no significant difference between the two groups. These data suggested that FA sensing based on mitochondrial oxidation was not activated in brain of $M$. amblycephala fed the HFD. This result was inconsistent with our previous findings in which the mRNA level in liver of $P P A R \alpha$ and CPT1 in $M$. amblycephala of the HFD group was significantly lower than that in the NFD group ${ }^{(42)}$. In that study, Lu et $a l .{ }^{(42)}$ held this view that the decline in expression of PPAR $\alpha$ and CPT1 was attributed to mitochondrial dysfunction caused by fat accumulation in liver. Different from liver, brain was a tissue for informational integration and almost no reports had shown that HFD could cause mitochondrial dysfunction in brain of fish. Facing higher circulating level of FA, brain tissue perhaps developed other adaptive strategies, such as strengthening the transport of FA. FAT (CD36) was the translocase responsible for transmembrane transport of $\mathrm{FA}^{(43)}$. In this research, the mRNA level of $F A T / C D 36$ and SREBP1C was significantly elevated owing to the higher dietary lipid content. Similar findings had also been reported in grass carp that the mRNA level of
FAT/CD36 was increased in fish fed HFD ${ }^{(44)}$. The data obtained in recent years confirmed that FA regulated appetite possibly by directly binding to FAT/CD36 and then activated SREBP1c ${ }^{(17,19)}$. However, Librán-Pérez et $a l .{ }^{(20)}$ had also reported that FA sensing based on FAT/CD36 in fish was more dependent on the presence of specific FA, such as oleate or octanoate, rather than global FA. In our opinion, the higher mRNA levels of $F A T / C D 36$ and SREBP1c observed in this experiment might be owing to the differences in FA composition between the HFD and NFD and the fact that fish fed a long-term HFD consumed more specific FA. Activation of sensor systems for FA by directly binding to FAT/CD36 was more dependent on specific FA, whereas appetite suppression caused by the HFD was independent of specific FA. Therefore, we hold that the decline in appetite caused by the HFD might be partly, not only, mediated by FA sensing based on FA transport. There were other factors involved in the HFD that suppressed appetite.

In addition to sensor systems for FA, the inflammatory factors were known to be other potential contributors to the appetite suppression of fish fed HFD. In mammals, suppression of appetite was a common and debilitating symptom that occured as a consequence of acute and chronic inflammation ${ }^{(27)}$. Our previous study found that the expression of $T N F-\alpha$ in the liver of M. amblycephala fed HFD was significantly increased, suggesting that chronic inflammation might be developed in the HFD group ${ }^{(35)}$. In this study, we systematically monitored the inflammatory response induced by long-term feeding of the HFD. The data showed that the mRNA levels of TNF- $\alpha$, IL- 6 and MCP-1 in the brain and the protein content of those inflammatory cytokines were significantly up-regulated in the HFD group. In addition, the NO content in plasma, considered as an inflammatory marker, was significantly increased in the HFD group. In fish, the up-regulation of the inflammatory factors mRNA expression might be related to signalling molecules for regulation of inflammatory gene transcriptions. One of the upstream signalling molecules of pro-inflammatory factors was $\mathrm{NF}-\kappa \mathrm{B}^{(45)}$. Once NF- $\kappa \mathrm{B}$ was activated, it induced the expression of multiple inflammatory mediators, including TNF- $\alpha$, IL- 6 and $\mathrm{MCP}-1^{(46)}$. I $\kappa \mathrm{B}$, a cytoplasmic protein, binded to $\mathrm{NF}-\kappa \mathrm{B}$ and masked the nuclear localisation sequence, thereby preventing the nuclear translocation ${ }^{(47)}$. It had been reported that the down-regulation of $\mathrm{I} \kappa \mathrm{B}$ gene expression stimulated nuclear translocation of NF- $\kappa \mathrm{B}$, thus evoking the transcription of $\mathrm{NF}-\kappa \mathrm{B}$ target genes ${ }^{(48)}$. Data in this research showed that long-term HFD facilitated $N F-\kappa B$ nuclear translocation to elevate proinflammatory factor expression by down-regulating the expression of $I \kappa B$ in brain. Those data in this experiment suggest that HFD feeding would leave $M$. amblycephala in longterm chronic inflammation. Studies in mammals showed that leptin was involved in the regulation of appetitive gene expression by inflammatory factors ${ }^{(27)}$. In this study, data showed that the mRNA expression of leptin in the brain and its protein context in plasma were significantly increased in fish fed the HFD. In fish, a similar result in grass carp had also been reported - that long-term feeding of HFD resulted in a significant increase in the mRNA level of leptin ${ }^{(4)}$. In mammals, one of the classic pathways by which leptin regulated appetite suppression was the activation of mTOR phosphorylated by 
$\mathrm{AKT}^{(29)}$. In this study, the mRNA levels of AKT and mTOR were increased in the HFD group than those in the NFD group. Similar results in rainbow trout had also been reported - that higher protein levels of AKT and mTOR were observed in fish fed a HFD ${ }^{(20)}$. Our data thus confirm that inflammation was a potential factor for appetite suppression induced by HFD through elevating the expression of leptin and then activating mTOR.

To further verify the effects of inflammatory cytokines on appetite neuropeptides in $M$. amblycephala, LPS was administered by intraperitoneal injection to induce an inflammatory response. The results showed that inflammation occurred at $6 \mathrm{~h}$ after injection and disappeared $48 \mathrm{~h}$ later. Similar results had also been reported in Eriocheir sinensis and M. amblycephala that inflammatory response reached its peak at 6-12 $\mathrm{h}$ after LPS injection $^{(35,49)}$. Concerning appetite, data in current study revealed that appetite was significantly suppressed in fish injected with LPS when the inflammatory response was processing, which was directly evidenced by a decrease in food intake and was further evidenced by the significantly decreased expression of $N P Y$ and the significantly elevated expression of CART. Furthermore, the mRNA levels of leptin and $m T O R$ were significantly increased after LPS injection. In rats, decreased food intake owing to LPS injection was associated with activation of mTOR, and blocking the mTOR pathway significantly attenuated appetite suppression induced by $\operatorname{LPS}^{(26)}$. These results suggested that inflammation-induced appetite suppression in $M$. amblycephala might be related to the molecular signalling of leptin-activated $m T O R$

In summary, the suppression of appetite was responsible for the reason that long-term HFD failed to promote the growth of M. amblycephala. The decline in appetite caused by high fat may be partly, not the only, mediated by sensor systems for FA based on FA transport. Besides, the inflammation was another potential contributor to the appetite suppression of fish fed a HFD. Results from this study implied inflammatory factors induced by HFD might be involved in the regulation of appetite by increasing the secretion of leptin and then activating mTOR.

\section{Acknowledgements}

The authors are grateful to members of Aquatic Animal Nutrition Group of Nanjing Agricultural University (CongCong Wang, Li Zhang, XiuFei Cao and JiaDai Liu) for assistance with sample collection.

This research was supported by the National Natural Science Foundation of China (G.-Z. J., grant no. 31502178), China Agriculture Research System (W.-B. L., grant no. CARS-45-14) and the Fundamental Research Funds for the Central Universities (G.-Z. J., grant no. KJQN201612).

The authors' contributions were as follows: Y.-J. D., G.-Z. J. and W.-B. L. designed the research; Y.-J. D. and W.-B. L. formulated the research question; Y.-J. D. and X.-Y. Y. conducted the research and analysed the data; and all authors contributed to writing the article and had primary responsibility for the final content. All authors read and approved the final manuscript.

The authors declare that there are no conflicts of interest.

\section{References}

1. Ortinau LC, Hoertel HA, Douglas SM, et al. (2014) Effects of high-protein vs. high-fat snacks on appetite control, satiety, and eating initiation in healthy women. Nutr J 13, 97.

2. Tantot F, Parkes SL, Marchand AR, et al. (2017) The effect of high-fat diet consumption on appetitive instrumental behavior in rats. Appetite 108, 203-211.

3. Furlong TM, Jayaweera HK, Balleine BW, et al. (2014) Bingelike consumption of a palatable food accelerates habitual control of behavior and is dependent on activation of the dorsolateral striatum. J Neurosci 34, 5012-5022.

4. Li A, Yuan X, Liang XF, et al. (2016) Adaptations of lipid metabolism and food intake in response to low and high fat diets in juvenile grass carp (Ctenopharyngodon idellus). Aquaculture 457, 43-49.

5. Du ZY, Liu YJ, Tian LX, et al. (2015) Effect of dietary lipid level on growth, feed utilization and body composition by juvenile grass carp (Ctenopharyngodon idella). Aquacult Nutr 11, 139-146.

6. Li X, Jiang Y, Liu W, et al. (2012) Protein-sparing effect of dietary lipid in practical diets for blunt snout bream (Megalobrama amblycephala) fingerlings: effects on digestive and metabolic responses. Fish Physiol Biochem 38, 529-541.

7. Tocher DR, Bell JG, Mcghee F, et al. (2003) Effects of dietary lipid level and vegetable oil on fatty acid metabolism in Atlantic salmon (Salmo salar L.) over the whole production cycle. Fish Physiol Biochem 29, 193-209.

8. Cai Z, Mai K \& Ai Q (2017) Regulation of hepatic lipid deposition by phospholipid in large yellow croaker. Br J Nutr 118, 999-1009.

9. Gélineau A, Corraze G, Boujard T, et al. (2001) Relation between dietary lipid level and voluntary feed intake, growth, nutrient gain, lipid deposition and hepatic lipogenesis in rainbow trout. Reprod Nutr Dev 41, 487-503.

10. Zhou Y, Liang XF, Yuan X, et al. (2013) Neuropeptide $\mathrm{Y}$ stimulates food intake and regulates metabolism in grass carp, Ctenopharyngodon idellus. Aquaculture 380-383, 52-61.

11. Kobayashi Y, Peterson BC \& Waldbieser GC (2008) Association of cocaine- and amphetamine-regulated transcript (CART) messenger RNA level, food intake, and growth in channel catfish. Comp Biochem Phys A 151, 219-225.

12. Zhou Y, Liang XF, Yuan X, et al. (2013) Neuropeptide Y stimulates food intake and regulates metabolism in grass carp, Ctenopharyngodon idellus. Aquaculture 380, 52-61.

13. Librán-Pérez M, Polakof S, López-Patiño MA, et al. (2012) Evidence of a metabolic fatty acid-sensing system in the hypothalamus and Brockmann bodies of rainbow trout: implications in food intake regulation. Am J Physiol Regul Integr Comp Physiol 302, R1340-R1350.

14. Gao S, Moran TH, Lopaschuk GD, et al. (2013) Hypothalamic malonyl-CoA and the control of food intake. Physiol Behav 122, $17-24$.

15. Migrenne S, Magnan C \& Cruciani-Guglielmacci C (2007) Fatty acid sensing and nervous control of energy homeostasis. Diabetes Metab 33, 177-182.

16. Tang Z, Sun C, Yan A, et al. (2013) Genes involved in fatty acid metabolism: molecular characterization and hypothalamic mRNA response to energy status and neuropeptide $\mathrm{Y}$ treatment in the orange-spotted grouper Epinephelus coioides. Mol Cell Endocrinol 376, 114-124.

17. Libránpérez M, Lópezpatiño MA, Míguez JM, et al. (2013) Oleic acid and octanoic acid sensing capacity in rainbow trout Oncorbynchus mykiss is direct in hypothalamus and Brockmann bodies. PLOS ONE $\mathbf{8}$, e59507. 
18. Librán-Pérez M, Otero-Rodiño C, Míguez JM, et al. (2014) Central administration of oleate or octanoate activates hypothalamic fatty acid sensing and inhibits food intake in rainbow trout. Physiol Behav 129, 272-279.

19. Soengas JL (2014) Contribution of glucose- and fatty acid sensing systems to the regulation of food intake in fish. A review. Gen Comp Endocr 205, 36-48.

20. Librán-Pérez M, Geurden I, Dias K, et al. (2015) Feeding rainbow trout with a lipid-enriched diet: effects on fatty acid sensing, regulation of food intake and cellular signaling pathways. J Exp Biol 218, 2610-2619.

21. Carrero JJ, Qureshi AR, Axelsson J, et al. (2007) Comparison of nutritional and inflammatory markers in dialysis patients with reduced appetite. Am J Clin Nutr 85, 695-701.

22. SchãBitz B, Pezeshki G, Pohl T, et al. (1995) Soluble interleukin-6 (IL-6) receptor augments central effects of IL-6 in vivo. FASEB J 9, 659-664.

23. Sergeyev V, Broberger C \& Hökfelt T (2001) Effect of LPS administration on the expression of POMC, NPY, galanin, CART and MCH mRNAs in the rat hypothalamus. Brain Res Mol Brain Res 90, 93-100.

24. Arruda AP, Milanski M, Romanatto T, et al. (2010) Hypothalamic actions of tumor necrosis factor alpha provide the thermogenic core for the wastage syndrome in cachexia. Endocrinology 151, 683-694.

25. Dudele A, Fischer CW, Elfving B, et al. (2015) Chronic exposure to low doses of lipopolysaccharide and high-fat feeding increases body mass without affecting glucose tolerance in female rats. Physiol Rep 3, 11-16.

26. Yue Y, Wang Y, Li D, et al. (2015) A central role for the mammalian target of rapamycin in LPS-induced mice anorexia. J Endocrinol 224, 37-47.

27. Dwarkasing JT, Marks DL, Witkamp RF, et al. (2016) Hypothalamic inflammation and food intake regulation during chronic illness. Peptides 77, 60-66.

28. Chu SC, Chen PN, Chen JR, et al. (2018) Role of hypothalamic leptin-LepRb signaling in NPY-CART-mediated appetite suppression in amphetamine-treated rats. Horm Behav 98, 173-182.

29. Mayamonteiro CM \& Bozza PT (2008) Leptin and mTOR: partners in metabolism and inflammation. Cell cycle 7, 1713-1717.

30. Cava AL \& Matarese G (2004) The weight of leptin in immunity. Nat Rev Immunol 4, 371-379.

31. Landman RE, Puder JJ, Xiao E, et al. (2003) Endotoxin stimulates leptin in the human and nonhuman primate. J Clin Endocrinol Metab 88, 1285-1291.

32. Li GG, Liang XF, Xie Q, et al. (2010) Gene structure, recombinant expression and functional characterization of grass carp leptin. Gen Comp Endocrinol 166, 117-127.

33. Gong N, Jönsson E \& Bjornsson BT (2015) Acute anorexigenic action of leptin in rainbow trout is mediated by the hypothalamic Pi3k pathway. J Mol Endocrinol 56, 227-238

34. Landgraf K, Schuster S, Meusel A, et al. (2017) Short-term overfeeding of zebrafish with normal or high-fat diet as a model for the development of metabolically healthy versus unhealthy obesity. BMC Physiol 17, 4.

35. Zhou M, Mi HF, Liu WB, et al. (2017) Molecular characterisation of tumour necrosis factor alpha and its potential connection with lipoprotein lipase and peroxisome proliferator-activated receptors in blunt snout bream (Megalobrama amblycephala). J Appl Genet 58, 1-11.

36. Kilkenny C, Browne W, Cuthill IC, et al. (2010) Animal research: reporting in vivo experiments: the ARRIVE guidelines. J Gene Med 12, 561-563.

37. $\mathrm{Xu}$ WN, Chen $\mathrm{DH}$, Chen QQ, et al. (2017) Growth performance, innate immune responses and disease resistance of fingerling blunt snout bream, Megalobrama amblycephala adapted to different berberine-dietary feeding modes. Fish Shellfish Immunol 68, 458-465.

38. Gélineau A, Bolliet V, Corraze G, et al. (2002) The combined effects of feeding time and dietary fat levels on feed intake, growth and body composition in rainbow trout. Aquat Living Resour 15, 225-230.

39. Zhou Y, Liang XF, Yuan X, et al. (2013) Neuropeptide Y stimulates food intake and regulates metabolism in grass carp, Ctenopharyngodon idellus. Aquaculture 380, 52-61.

40. Liu L, Liang XF, Li J, et al. (2014) Feed intake, feed utilization and feeding-related gene expression response to dietary phytic acid for juvenile grass carp (Ctenopharyngodon idellus). Aquaculture 424-425, 201-206.

41. Lu KL, Zhang DD, Wang LN, et al. (2016) Molecular characterization of carnitine palmitoyltransferase IA in Megalobrama amblycephala and effects on its expression of feeding status and dietary lipid and berberine. Comp Biochem Phys B 191, 20-25.

42. Lu KL, Xu WN, Wang LN, et al. (2014) Hepatic $\beta$-oxidation and regulation of carnitine palmitoyltransferase (CPT) I in blunt snout bream Megalobrama amblycephala fed a high fat diet. PLOS ONE 9, e93135.

43. Zhou JS, Li RW, Lin YQ, et al. (2018) LCFA uptake and FAT/ CD36: molecular cloning, tissue expression and mRNA expression responses to dietary oil sources in grass carp (Ctenopharyngodon idellus). J Appl Anim Res 46, 572-582.

44. Tian J, Liu W, Gao W, et al. (2017) Molecular cloning and gene/protein expression of FAT/CD36 from grass carp (Ctenopharyngodon idella) and the regulation of its expression by dietary energy. Fish Physiol Biochem 43, 875-888.

45. Dong YW, Jiang WD, Liu Y, et al. (2017) Threonine deficiency decreased intestinal immunity and aggravated inflammation associated with NF-kappa B and target of rapamycin signalling pathways in juvenile grass carp (Ctenopharyngodon idella) after infection with Aeromonas hydrophila. Br J Nutr 118, 92-108.

46. Wang T, Yang B, Ji R, et al. (2017) Omega-3 polyunsaturated fatty acids alleviate hepatic steatosis-induced inflammation through Sirt1-mediated nuclear translocation of NF-кB p65 subunit in hepatocytes of large yellow croaker (Larmichthys crocea). Fish Shellfish Immunol 71, 76-82.

47. Correa RG, Matsui T, Tergaonkar V, et al. (2005) Zebrafish IкB

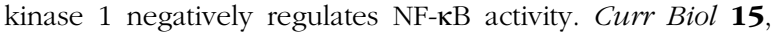
1291-1295.

48. Yu M, Chen J, Bao Y, et al. (2018) Genomic analysis of NF-kB signaling pathway reveals its complexity in crassostrea gigas. Fish Shellfish Immunol 72, 510-518.

49. Li S, Jia Z, Li X, et al. (2014) Identification and expression analysis of lipopolysaccharide-induced TNF-alpha factor gene in Chinese mitten crab Eriocheir sinensis. Fish Shellfish Immunol 38, 190-195.

50. Xu WN, Qian Y, Li XF, et al. (2017) Effects of dietary biotin on growth performance and fatty acids metabolism in blunt snout bream, Megalobrama amblycephala, fed with different lipid levels diets. Aquaculture 479, 790-797.

51. Pan W, Miao L, Lin Y, et al. (2017) Regulation mechanism of oxidative stress induced by high glucose through Pi3k/Akt/ Nrf2 pathway in juvenile blunt snout bream (Megalobrama amblycephala). Fish Shellfish Immunol 70, 66-75.

52. Xu C, Liu WB, Zhang DD, et al. (2018) Interactions between dietary carbohydrate and metformin: implications on energy sensing, insulin signaling pathway, glycolipid metabolism and glucose tolerance in blunt snout bream Megalobrama amblycephala. Aquaculture 483, 183-195. 